\title{
EL VOLUNTARIADO UNIVERSITARIO COMO ÁMBITO DE APRENDIZAJE SERVICIO Y EMPRENDIMIENTO SOCIAL. UN ESTUDIO DE CASO
}

\author{
RICARDO GAETE QUEZADA*
}

\begin{abstract}
RESUMEN
El objetivo del estudio es analizar la interacción que se genera entre el voluntariado universitario, el aprendizaje servicio y el emprendimiento social para el caso de las universidades de la ciudad de Antofagasta, desde la perspectiva del paradigma interpretativo-hermenéutico mediante un enfoque cualitativo y la entrevista semiestructurada como técnica de recolección de datos y a los procedimientos de la teoría fundamentada para analizar los resultados. En el caso estudiado, el voluntariado universitario es una iniciativa mayoritariamente de origen estudiantil, desarrollado para ejecutar acciones en beneficio de la comunidad, así como para fortalecer una mayor sensibilidad social y ciudadana de los estudiantes respecto de las problemáticas y necesidades de los habitantes más vulnerables de la ciudad. Así, el voluntariado universitario se transforma en un ámbito interesante para fortalecer los procesos de formación universitaria como expresión de un aprendizaje servicio, pero también como un espacio de fortalecimiento de los procesos de emprendimiento social, que trasciendan el contexto universitario y repercutan positivamente en el largo plazo en la comunidad local.
\end{abstract}

PALABRAS CLAVE: JÓVENES UNIVERSITARIOS, RESPONSABILIDAD SOCIAL UNIVERSITARIA, UNIVERSIDADES CHILENAS

* Académico e investigador del Departamento de Ciencias Sociales de la Universidad de Antofagasta, Doctor en Procesos de Cambio Social y Magíster en Gestión Pública. E-Mail: ricardo.gaete@uantof.cl.

El artículo presenta resultados pertenecientes al proyecto FONDECYT de Iniciación a la Investigación $\mathrm{N}^{\mathrm{o}} 11121144$, denominado «Una mirada a la responsabilidad social universitaria en la ciudad de Antofagasta». 


\title{
VOLUNTARIADO UNIVERSITÁRIO COMO ÂMBITO DA APRENDIZAGEM SERVIÇO E EMPREENDIMENTO SOCIAL. UM ESTUDO DE CASO
}

\begin{abstract}
RESUMO
O objetivo do estudo é analisar a interação gerada entre o voluntariado universitário, a aprendizagem serviço e o empreendimento social no caso das universidades da cidade de Antofagasta, da perspectiva do paradigma hermenêutico (interpretativo) mediante um enfoque qualitativo e a entrevista semi-estruturada como técnica de coleta de dados e os procedimentos da teoria fundamentada para analisar os resultados. No caso estudado, o voluntariado universitário é uma iniciativa que provêm fundamentalmente dos estudantes, desenvolvido para realizar ações em benefício da comunidade, assim como para fortalecer uma maior sensibilidade social e cidadã dos estudantes sobre as problemáticas e necessidades dos habitantes mais vulneráveis da cidade. Assim, o voluntariado universitário se torna um âmbito interessante para fortalecer os processos de formação universitária como expressão de uma aprendizagem serviço, mas também como um espaço de fortalecimento dos processos de empreendimento social, transcendendo o contexto universitário e repercutindo positivamente a longo prazo na comunidade local.
\end{abstract}

\section{PALAVRAS CHAVE: JOVENS UNIVERSITÁRIOS, RESPONSABILIDADE SOCIAL UNIVERSITÁRIA, UNIVERSIDADES CHILENAS}

\section{UNIVERSITY VOLUNTEERING AS A FIELD OF SERVICE LEARNING AND SOCIAL ENTREPRENEUSHIP: A CASE STUDY}

\begin{abstract}
The present study aims at analyzing the interaction generated among university volunteering, service learning and social entrepreneurship in the universities of Antofagasta from an interpretive-hermeneutic paradigm perspective. The data was collected through a qualitative approach, with semi-structured interview as the main data collection technique, and using methods from the grounded theory for data analysis. The results obtained describe university volunteering as mostly a student initiative, mainly developed to take actions both to benefit the community and to encourage social and civic sensitivity in students regarding the problems and needs of the most vulnerable people in Antofagasta. University volunteering, thus, becomes fundamental to strengthen the process of university education as an expression of service learning, but also as a space for strengthening social business processes that transcend the university context and impact positively over the long term within the local community.
\end{abstract}

KEY WORDS: UNIVERSITY STUDENTS, UNIVERSITY SOCIAL RESPONSIBILITY, CHILEAN UNIVERSITIES 


\section{INTRODUCCIÓN}

EL PRESENTE TRABAJO EXPONE una parte de los resultados del proyecto de iniciación a la investigación de FONDECYT Nº11121144, que estudia el concepto de Responsabilidad Social Universitaria (RSU) para el caso de la ciudad de Antofagasta en Chile. En este artículo, se analizan específicamente los aspectos relacionados con el concepto de voluntariado universitario como tópico emergente, en cuanto a las conexiones que se generan con el aprendizaje servicio y el emprendimiento social como una parte importante del comportamiento socialmente responsable de las universidades, especialmente en las funciones de docencia universitaria y vinculación con el medio. Por ello, en la primera parte del trabajo se analizan los principales elementos teóricos de los tres conceptos centrales de este trabajo, especialmente desde la perspectiva de identificar las posibles interacciones y complementos entre sí. La literatura muestra un debate interesante sobre las posibles interacciones entre el voluntariado universitario y el aprendizaje servicio.

En la segunda parte del trabajo, se analizan los principales puntos de vista y experiencias de los dirigentes estudiantiles universitarios recogidos mediante entrevistas semiestructuradas, para conocer su comprensión del concepto de RSU así como las buenas prácticas que permiten evidenciar dicho comportamiento organizacional o individual, que para el caso de los entrevistados se concentra en sus experiencias relacionadas con las actividades de voluntariado universitario.

Los resultados obtenidos en este caso de estudio ofrecen elementos que permiten proporcionar un triple impacto del voluntariado para el concepto de RSU: fortalece la sensibilidad social y ciudadana de los estudiantes, permite que los estudiantes pongan al servicio de la comunidad sus conocimientos y capacidades para atender alguna necesidad social configurando procesos de aprendizaje servicio no generados desde el plan curricular, y finalmente, favorece el desarrollo de iniciativas que se proyectan en el tiempo más allá del ámbito universitario para propiciar un cambio social que permita un desarrollo más sustentable y justo en la sociedad.

\section{REVISIÓN DE LA LITERATURA: EL VOLUNTARIADO UNIVERSITARIO}

Desde una perspectiva global, según el Informe del Programa de Voluntarios de las Naciones Unidas (2011:4) se señala que determinar 
qué es el voluntariado no resulta una tarea sencilla, ya que existe una gran cantidad de definiciones disponibles en la literatura, por lo que recomiendan identificar tres grandes criterios en las acciones de voluntariado:

- Voluntad libre: la acción debe llevarse a cabo de forma voluntaria, de acuerdo con la propia voluntad libremente expresada de la persona, y no como una obligación impuesta por una ley, un contrato o un requisito académico.

- Motivación no pecuniaria: la acción no debe llevarse a cabo primordialmente para la obtención de una recompensa económica. El reembolso de gastos o estipendios, así como los pagos en especie como los asociados a los gastos de transporte y manutención, deben justificarse de forma adecuada.

- Beneficio para otros: la acción debe perseguir el bien común y favorecer de forma directa o indirecta a personas ajenas a la familia o el hogar del voluntario, o bien realizarse en favor de una determinada causa, incluso si la persona que actúa como voluntaria también resulta beneficiada.

Estos aspectos señalados por las Naciones Unidas abarcan un espectro amplio de formas de expresión del voluntariado (ver cuadro 1), incluyendo tanto el voluntariado desarrollado al alero de alguna institución formal, así como el voluntariado realizado por personas que no pertenecen a ningún contexto oficial en cuanto a su carácter organizativo. No obstante, investigaciones como la de Ríos (2004) descartan como expresiones del voluntariado a las acciones de ayuda a terceros desarrolladas individualmente fuera de una institución o marco organizativo formal específico, especialmente cuando se dirigen a familiares, parientes y amigos, confirmando que la definición del voluntariado no es una tarea fácil.

Evidentemente, las expresiones del voluntariado descritas en el Cuadro 1 no son las únicas existentes, así por ejemplo en la investigación de Ríos (2004) sobre el involucramiento de estudiantes universitarios en acciones de voluntariado, se consideran a la ayuda a terceros (filantropía), distintos tipos de participación: en asociaciones de interés (centros de alumnos y federaciones de estudiantes), en actividades culturales, en causas y campañas y en movimientos o partidos políticos. Algunos de los ejemplos señalados anteriormente, en cierta medida nos recuerdan las variables analizadas en la investigación sobre capital social a nivel mundial desarrollada por Putnam (2003), 
demostrando el gran valor e impacto que tiene para la sociedad la ejecución de acciones de voluntariado.

\section{CUADRO 1: FORMAS DE EXPRESIÓN DEL VOLUNTARIADO}

\begin{tabular}{|c|l|}
\hline Tipo & Descripción \\
\hline $\begin{array}{c}\text { Prestación de un } \\
\text { servicio }\end{array}$ & $\begin{array}{l}\text { La provisión de un servicio a un tercero. Por lo general dicha } \\
\text { provisión tiene lugar a través de estructuras existentes, que } \\
\text { abarcan una amplia gama de esferas sociales, culturales y de } \\
\text { desarrollo. }\end{array}$ \\
\hline $\begin{array}{c}\text { Ayuda mutua o } \\
\text { autoayuda }\end{array}$ & $\begin{array}{l}\text { Consiste en que personas que comparten unas mismas necesi- } \\
\text { dades, problemas o intereses unen sus fuerzas para darles } \\
\text { respuesta. Los miembros del grupo también se benefician del } \\
\text { proceso. }\end{array}$ \\
\hline Participación & $\begin{array}{l}\text { Desarrollo de campañas e iniciativas que buscan provocar o } \\
\text { impedir un cambio a nivel local o a pequeña escala, pudiendo } \\
\text { en ocasiones dar origen a movimientos sociales más amplios a } \\
\text { escala nacional o global. }\end{array}$ \\
\hline
\end{tabular}

Fuente: elaboración propia, basado en Programa de Voluntarios de Naciones Unidas (2011).

De esta manera, Soler (2007:25) define el concepto de voluntariado como «un conjunto de personas que ha adquirido una conciencia solidaria fundamentada en una visión crítica de la realidad y en su derecho como ciudadano, desarrollando actividades de forma altruista y solidaria, basadas en su libre decisión, en un compromiso con el marco organizativo que le facilita un proceso formativo adecuado. La finalidad última de su colaboración es la transformación de la realidad social, con unos ideales que aspiran a crear un mundo más solidario, justo y pacífico». Esta definición confirma en buena medida que las acciones de voluntariado están estrechamente relacionadas con una conducta pro social de los voluntarios, como expresión de una conciencia sobre la realidad y desigualdad social imperante en la sociedad, situación que según Jara y Vidal (2010) es lo que permite desarrollar un mayor sentido de la responsabilidad social dentro de la comunidad.

La definición expuesta anteriormente también está directamente relacionada con una evolución del concepto tradicional del voluntariado hacia una concepción actual, que agrega nuevas características a dicho término, como se observa en el cuadro 2. 
CUADRO 2: DIFERENCIAS ENTRE EL VOLUNTARIADO TRADICIONAL Y ACTUAL

\begin{tabular}{|c|c|c|}
\hline & Voluntariado tradicional & Voluntariado actual \\
\hline Población objetivo & $\begin{array}{l}\text { Dirigido a los pobres y } \\
\text { menesterosos. }\end{array}$ & $\begin{array}{l}\text { Dirigido a cualquier persona } \\
\text { miembro de la comunidad. }\end{array}$ \\
\hline $\begin{array}{c}\text { Tipo de } \\
\text { intervención }\end{array}$ & $\begin{array}{l}\text { El individuo es un mero } \\
\text { receptor de la ayuda. }\end{array}$ & $\begin{array}{l}\text { Implicación de la comuni- } \\
\text { dad para resolver sus } \\
\text { dificultades. }\end{array}$ \\
\hline $\begin{array}{l}\text { Consecuencias de } \\
\text { la intervención }\end{array}$ & Pasividad del usuario. & Participación activa. \\
\hline $\begin{array}{c}\text { Objetivo de la } \\
\text { ayuda }\end{array}$ & $\begin{array}{l}\text { La tarea es un fin en sí } \\
\text { misma. }\end{array}$ & $\begin{array}{l}\text { Únicamente es un medio } \\
\text { para el voluntario. }\end{array}$ \\
\hline Marco de actuación & Espontáneo. & Organizativo. \\
\hline Capacitación & $\begin{array}{l}\text { Con buena voluntad es } \\
\text { suficiente. }\end{array}$ & Formación adecuada. \\
\hline Motivaciones & $\begin{array}{l}\text { Motivos religiosos o } \\
\text { morales, fundamentalmente. }\end{array}$ & Pluralidad de motivos. \\
\hline $\begin{array}{l}\text { Reconocimiento } \\
\text { legal }\end{array}$ & Sin control normativo. & Sujeto a normativa jurídica. \\
\hline
\end{tabular}

Fuente: Soler (2007:55).

Según lo planteado en el cuadro 2, actualmente el voluntariado se orienta hacia una mayor profesionalización de sus actividades, pero también debe sustentarse en la incidencia política y ciudadana que deben adquirir las personas que desarrollan esta actividad, con el propósito de combinar más equilibradamente la entrega de la ayuda con una mayor concienciación sobre la relevancia de generar cambios en la sociedad, para que las problemáticas o necesidades sociales que son atendidas mediante el voluntariado sean resueltas a largo plazo de manera definitiva por el Estado, a través de políticas públicas más efectivas que permitan superar las injusticias y las desigualdades sociales. Por esto, Jara y Vidal (2010) afirman que el trabajo voluntario debe generar una concienciación transformadora, tanto en los voluntarios como en las personas de la sociedad hacia las cuales se dirigen las acciones de voluntariado.

Ahora bien, cuando se analiza el voluntariado al interior de las universidades, Arias (2008:30) señala que «hace alusión no solo al voluntariado que tiene como protagonistas de la acción a los jóvenes universitarios, sino también a toda la política universitaria y al conjunto de actividades llevadas a cabo desde la comunidad universitaria orientadas hacia este campo. Se trata, por lo tanto, de un concepto amplio que abarca y engloba a toda la comunidad universitaria y a todos los ámbitos que como universidad le son propios». Lo anterior, 
hace necesario transformar a las actividades de voluntariado universitario en un aspecto integral del quehacer universitario y no como un hecho aislado o circunstancial, existiendo diferentes modalidades que el voluntariado asume en el ámbito universitario:

Formal, a través de la denominada proyección social que la universidad realiza como institución; o informal, cuando son los estudiantes los que se reúnen para desplegar esta actividad a través de sus propias organizaciones estudiantiles (Vallaeys, s/f).

Orientado a cubrir necesidades asistenciales de sectores desfavorecidos sin que los voluntarios pongan en práctica sus conocimientos y capacidades específicas; u orientado a que los voluntarios apliquen los conocimientos y habilidades adquiridas más allá de una concepción asistencialista, promoviendo el desarrollo más sustentable de la sociedad (Méndez, 2009).

Vinculado con instituciones que trabajen con voluntarios pero que se encuentran fuera de la universidad como una ONG; o desarrollado dentro de la propia universidad a través de proyectos de extensión para promover el desarrollo profesional de los estudiantes (Silva et al., 2013).

Si bien las modalidades de voluntariado universitario expuestas anteriormente son convergentes con lo señalado por Naciones Unidas según lo expuesto anteriormente en el cuadro 1, según Vallaeys (s/f) el verdadero desafío de las universidades socialmente responsables respecto del voluntariado universitario, radica no solo en elegir qué tipo de voluntariado promover desde su interior, sino que muy especialmente ser capaces de articularlo con la formación y la investigación, para lograr que los voluntarios universitarios sean también ciudadanos plenamente consciente respecto de las razones por las cuales se originan los problemas sociales, para intentar incidir en la búsqueda de soluciones definitivas que permitan superar dichas desigualdades dentro de la sociedad.

Asimismo, Arias (2008:24) afirma que cuando la articulación del voluntariado con la formación es correctamente gestionada dentro de las universidades «se convierte en los campus universitarios en un instrumento educativo y formativo que complementa la formación que los estudiantes reciben en las aulas y que no estaba contemplada de esta manera hasta este momento. Así, los años de estudios universitarios se convierten también para los jóvenes en años de aprendizaje social, durante los cuales se fomenta la actuación del universitario en la transformación de la sociedad». Por ejemplo, investigaciones como 
las de Weerts et al., (2010) sobre los impactos no monetarios de la participación de los estudiantes frente a las donaciones caritativas, muestran beneficios interesantes de la interacción entre la incidencia política y el voluntariado, destacando en el primer aspecto a la generación de una mayor incidencia política de los estudiantes sobre autoridades regionales o locales, mientras que en lo relativo al voluntariado, se destacan las tutorías a los nuevos estudiantes, reclutamiento de voluntarios para eventos especiales, entre otras.

Asimismo, los procesos de voluntariado otorgan a la universidades la oportunidad de formar a sus estudiantes en valores tan importantes como el respeto, la tolerancia o la empatía con el otro, lo que les permitirá «asegurarse de que sus estudiantes han adquirido la sensibilidad suficiente para que valoren la dimensión humana que acompaña cada problema y así poder entender mejor para poder decidir mejor y más justamente» (Arias, 2008:33). Otros beneficios importantes para los estudiantes que destinan tiempo al desarrollo de actividades de voluntariado universitario es que cambian su perspectiva y sensibilidad sobre su responsabilidad cívica (Lester et al., 2012).

\section{APRENDIZAJE SERVICIO Y VOLUNTARIADO EN EL ÁMBITO UNIVERSITARIO}

Desde hace más de dos décadas, los programas de aprendizaje servicio han mostrado un crecimiento sostenido y sustancial en la educación superior a nivel mundial (Gray, 2000; Mooney y Edwards, 2001; Sedlak et al., 2003; Arratia, 2008; Rodríguez, 2014).

De acuerdo con Rodríguez (2014:96) el aprendizaje servicio implica «una forma de educación experiencial en la que los estudiantes se comprometen en actividades de ayuda a la comunidad al tiempo que facilita el aprendizaje de una asignatura y el desarrollo de competencias profesionales», transformándolo en una estrategia de enseñanza que debiera incrementar su presencia en los procesos de formación universitaria para romper con lo que Freire (2008:73) denomina como la «educación bancaria»; es decir, «el acto de depositar en el cual los educandos son los depositarios y el educador quien deposita», que hace que los estudiantes universitarios sean sujetos pasivos de su propio proceso de formación donde predomina la clase de los docentes universitarios de tipo expositiva, teórica y absolutamente desconectada del entorno. 
Por ello, el aprendizaje servicio según Martínez (2010:18) constituye una innovación docente en el ámbito universitario porque «introducen como novedad que el aprendizaje del estudiante se construya en un contexto de necesidades reales del entorno, tratando de mejorarlo. Es decir, constituyen una innovación en relación al aprendizaje en la universidad, y a la vez añaden a los objetivos clásicos de la formación universitaria otros que relacionan la actividad de aprendizaje del estudiante con su formación ciudadana», ratificando con ello la relevancia que debe tener para las universidades incluir en los procesos formativos a la sociedad y sus necesidades rompiendo la pasividad de los estudiantes en su propio proceso formativo, como recomienda Paulo Freire.

En síntesis, algunos beneficios de la utilización del aprendizaje servicio (Bringle y Hatcher, 1996; Sedlak et al., 2003; Arratia; 2008; Rodríguez, 2014) como método de enseñanza en el ámbito universitario son:

- Promueve una mayor formación práctica reelaborando los contenidos teóricos para hacerlos más pertinentes para el contexto social y económico.

- Fortalece la formación en valores en aspectos como prosocialidad, responsabilidad social, solidaridad, pertenencia de la actividad profesional - entre otros - ayudando a la formación para la ciudadanía de los profesionales.

- Favorece la vinculación con la comunidad puesto que la intervención surge de la demanda explícita de la sociedad y promueve la intervención de carácter profesional sobre una problemática social real.

$\mathrm{Al}$ analizar las posibles conexiones entre el aprendizaje servicio y el voluntario universitario, existen planteamientos en la literatura que advierten que tal relación no es factible. Martínez (2010:17) señala que los procesos de aprendizaje servicio «no deben confundirse con la prestación de servicios voluntarios. Deben tener relevancia y reconocimiento en términos académicos — deben suponer aprendizaje académico en los estudiantes - y deben contribuir a la mejora de la calidad de vida y del nivel de inclusión social en la población», identificando como un aspecto diferenciador entre ambos la existencia de un interés académico en el desarrollo de las actividades de aprendizaje servicio. En el cuadro 3, se presentan algunas diferencias entre ambos procesos. 
CUADRO 3: DIFERENCIAS ENTRE APRENDIZAJE SERVICIO Y VOLUNTARIADO Y SERVICIO COMUNITARIO

\begin{tabular}{|l|l|}
\hline \multicolumn{1}{|c|}{ Aprendizaje servicio } & Voluntariado y servicio comunitario \\
\hline $\begin{array}{l}\text { Enfoque pedagógico-solidario y meto- } \\
\text { dología de enseñanza-aprendizaje. }\end{array}$ & Enfoque pedagógico-solidario. \\
\hline $\begin{array}{l}\text { Objetivos de servicio y objetivos de } \\
\text { aprendizaje. }\end{array}$ & Objetivos de servicio. \\
\hline $\begin{array}{l}\text { Formación profesional y formación } \\
\text { ciudadana. }\end{array}$ & Formación ciudadana. \\
\hline $\begin{array}{l}\text { Requiere fases de preparación, acción y } \\
\text { reflexión. }\end{array}$ & Comprende la actividad misma. \\
\hline $\begin{array}{l}\text { Existen normas establecidas para el } \\
\text { desempeño del servicio. }\end{array}$ & No existen normas establecidas. \\
\hline $\begin{array}{l}\text { Requiere planteamiento anticipado a la } \\
\text { fecha del servicio. }\end{array}$ & Puede ocurrir en cualquier momento. \\
\hline $\begin{array}{l}\text { Debe realizarse con la supervisión } \\
\text { adecuada de un adulto. }\end{array}$ & $\begin{array}{l}\text { Puede realizarse sin supervisión de un } \\
\text { adulto. }\end{array}$ \\
\hline Certificado de acreditación del servicio. & No es un requisito. \\
\hline
\end{tabular}

Fuente: basado en Rodríguez (2014:97).

Pese a lo planteado en el cuadro 3, también existen en la literatura planteamientos que señalan que el aprendizaje servicio implica una evolución en términos pedagógicos, que establece una conexión intencionada con el voluntariado universitario como un ámbito importante para desarrollar procesos de formación universitaria de carácter más experiencial (Parker-Gwin, 1996; Mooney y Edwards, 2001).

Trilla (2009) en su intento por establecer una genealogía del aprendizaje servicio identifica tres vías generadoras del mismo, en la segunda de las cuales, denominada «la vía del servicio», se genera una vinculación entre el voluntariado y el aprendizaje servicio a partir de la existencia de un impulso ético y social, expresado en la voluntad de contribuir para que la sociedad mejore mediante acciones de servicio desinteresadas como las de voluntariado, logrando que las personas que las realicen aprendan sirviendo. No obstante, según el propio Trilla las personas que desarrollen las actividades de voluntariado deben poseer las capacidades, conocimientos y destrezas necesarias para desarrollar dichas acciones de servicio en beneficio de la sociedad. Asimismo, Batlle (2009) afirma que los movimientos sociales y de voluntariado desarrollan sus acciones de servicio de manera solidaria y desinteresada en favor de la comunidad, las que pueden estar enfocadas desde prioridades éticas, políticas o pedagógicas para realizarlas. 
Es en ese último aspecto señalado anteriormente que encontramos el fundamento para afirmar que es posible conectar los procesos de aprendizaje servicio con el voluntariado universitario, cuando las acciones en beneficio de la comunidad se inician desde el servicio a través del voluntariado de los estudiantes, iniciativas que además son desinteresadas porque nacen del propio interés de los futuros profesionales universitarios por contribuir a mejorar a la comunidad, colocando al servicio de la misma aquellas capacidades y conocimientos adquiridos en las aulas universitarias; o sea, existe un impulso ético y social de los estudiantes que los mueve siendo además una interesante instancia para desarrollar valores socialmente responsable y de liderazgo social (Parker-Gwin, 1996). Las acciones de voluntariado universitario se transforman en un espacio de aprendizaje para ellos, lo que les permite complementar su formación teórica proporcionada por la universidad, con la puesta en práctica de sus habilidades al servicio de la comunidad para intentar producir un cambio social, paralelamente con un aumento de su propia conciencia ciudadana y social respecto de su entorno.

\section{DEL VOLUNTARIADO UNIVERSITARIO AL EMPRENDIMIENTO SOCIAL COMO DESAFÍO PARA LA RSU}

El concepto de emprendimiento social posee una creciente presencia en la literatura, existiendo una gran cantidad de definiciones sobre el mismo así como experiencias internacionales de gran reconocimiento y prestigio tales como Ashoka o Grameen Bank (Guzmán y Trujillo, 2008; Gatica, 2011; Bargsted, 2013). Algunas de las razones que explican la complejidad que reviste precisar su definición, están relacionadas con el hecho de que el emprendimiento social apunta a obtener un cambio social mediante el desarrollo de iniciativas que funcionan con una orientación empresarial, pero que persiguen objetivos sociales y medioambientales que priman por sobre los objetivos económicos (Guzmán y Trujillo, 2008; Gatica, 2011; Blanco et al., 2012; Curto, 2012; Bargsted, 2013).

Blanco et al., (2012:49) afirman que el emprendedor social «pretende ofrecer una alternativa de vida a su sociedad, una alternativa que favorezca a la mayoría, pero que al mismo tiempo permita el desarrollo personal de cada individuo [...] apela a la conciencia de las gentes para que descubran más allá del yo y el ellos: el nosotros». Además, estos autores señalan que si bien el emprendedor social comparte al- 
gunas características con otro tipo de emprendedores de tipo más empresarial, identifican como competencias distintivas del perfil del emprendedor social: la capacidad para tomar conciencia de la existencia de una desigualdad, la necesidad de existencia de una justicia social y la toma de decisiones a partir de una posición empática generada por la preocupación por los demás.

Sin embargo, para Guzmán y Trujillo (2008:110) la creación de valor social sostenible es un elemento diferenciador del emprendimiento social respecto de las acciones caritativas o de beneficencia, buscando generar soluciones concretas y definitivas para problemáticas sociales como por ejemplo, el analfabetismo, la drogadicción o la contaminación ambiental, lo que requiere a juicio de los autores la introducción de cambios sociales profundos, definiendo el emprendimiento social como «un tipo específico de emprendimiento que busca soluciones para problemas sociales a través de la construcción, evaluación y persecución de oportunidades que permitan la generación de valor social sostenible, alcanzando equilibrios nuevos y estables en relación con condiciones sociales, a través de la acción directa llevada a cabo por organizaciones sin ánimo de lucro, empresas y organismos gubernamentales».

En el ámbito universitario, el desarrollo de emprendimientos académicos o científicos especialmente relacionados con los procesos de transferencia del conocimiento e innovación, se están transformando en una nueva forma de expresión dentro del quehacer de las universidades a nivel mundial, como lo demuestra por ejemplo la experiencia acumulada en el Tecnológico de Monterrey en México (González y López, 2012). También, existen un sinnúmero de buenas prácticas relacionadas con iniciativas para generar emprendimientos e innovación desde las instituciones universitarias, entre las que el emprendimiento social es una instancia que comienza a manifestarse al interior de las universidades (Cicero et al., 2012). Asimismo, los estudios e investigaciones sobre las características y motivaciones emprendedoras en los estudiantes universitarios igualmente son un ámbito relevante a desarrollar dentro de las universidades, para fomentar la cultura y actitud emprendedora tanto en la propia universidad como en el resto de la sociedad (Rodríguez y Prieto, 2009; Martín y Lima, 2013).

A nuestro juicio, un desafío importante para las universidades socialmente responsables radica en generar las condiciones necesarias para que las actividades de voluntariado universitario que se gestan al interior de las instituciones universitarias, puedan transformarse en 
verdaderos emprendimientos sociales que creen dentro de la sociedad un conjunto de organizaciones que se preocupen por lograr un verdadero cambio social, que se traduzca en una sociedad más sustentable, justa y equitativa que intente eliminar las desigualdades sociales actualmente existentes.

\section{Metodología}

El artículo pertenece al proyecto FONDECYT de Iniciación a la investigación $\mathrm{N}^{\circ} 11121144$ denominado «Una mirada a la responsabilidad social universitaria en la ciudad de Antofagasta». La investigación se desarrolla desde la perspectiva del paradigma interpretativo-hermenéutico, por lo que se intenta comprender el concepto de RSU desde la mirada dialógica y simbólica del significado que las partes interesadas en el quehacer universitario atribuyen al mismo, para lo cual se utiliza un diseño de investigación cualitativo.

En cuanto al método de investigación, se utiliza el estudio de caso de tipo único e instrumental (Stake, 2007) para examinar de manera detallada, comprehensiva, sistemática y en profundidad a los diferentes aspectos relacionados con la RSU, utilizando para ello la experiencia y grado de conocimiento acumulado por las partes interesadas de las dos universidades pertenecientes al Consejo de Rectores de las Universidades Chilenas (CRUCH) existentes en la ciudad de Antofagasta que poseen su casa central en dicha localidad.

La técnica de recolección de la información es la entrevista semiestructurada desarrollada individualmente, en un solo encuentro y enfocadas en una sola temática, con partes interesadas internas y externas de las universidades de la ciudad de Antofagasta. El entrevistador proporcionará el mismo estímulo o planteamiento inicial a cada entrevistado sobre la problemática estudiada, utilizándose una pauta de cotejo con los tópicos relevantes para la investigación, y solamente en aquellos casos en los que el respectivo entrevistado no incluya dentro de su intervención alguno de dicho temas considerados en la pauta de cotejo, solo entonces se procederá a formular la pregunta respectiva sobre dicha temática ausente en el relato del entrevistado (Alonso, 2003; Corbetta, 2003).

En cuanto a los sujetos a entrevistar, se utiliza una muestra intencional de tipo teórica que prioriza la representatividad sustantiva por sobre aquella de tipo estadística, dado que lo que se intenta es incluir a los actores sociales de interés en cuanto al discurso que reproducen 
con el propósito de «recoger la información más relevante para el concepto o teoría buscada» (Ruiz Olabuénaga, 2007:64), intentando saturar los lugares de enunciación; es decir, cuando los datos que surjan de las entrevistas comiencen a repetirse y no aporten nuevos elementos al análisis del tema investigado.

Una vez transcritas las entrevistas para desarrollar el análisis e interpretación de los datos se seguirán los procedimientos establecidos por la Teoría Fundamentada en los datos (Grounded Theory) apoyados por el software Atlas $\mathrm{Ti}$, mediante el siguiente procedimiento: identificación de los incidentes para asignarle códigos (Microanálisis); comparación de los códigos sustantivos, conceptos y categorías (Codificación); y la integración de estos elementos en una teoría que se elabora a partir de los datos que permitan construir hipótesis teóricas relativa a la identificación de la categoría central.

En el caso específico del microanálisis como expresión de una primera reducción de los textos analizados, es importante determinar lo que Bardin (2002) denomina como la «unidad de registro» para identificar la extensión de los segmentos que serán utilizados para ubicar a los incidentes de los textos, lo cual en el caso de la teoría fundamentada puede desarrollarse el análisis por palabras, líneas, oraciones o párrafos (Charmaz, 2006; Strauss y Corbin, 2002). En nuestra investigación, se ha optado por utilizar como unidad de registro a las oraciones o párrafos que encierren algún significado sobre el concepto de RSU.

\section{Resultados}

A partir de los discursos de las diferentes partes interesadas de las universidades incluidas en nuestro estudio de caso recogidas en las entrevistas, considerando los planteamientos de la Teoría Fundamentada es posible identificar algunas categorías, conceptos y códigos que están relacionados con el concepto de RSU, como se observa en el cuadro 4.

Es importante aclarar que lo expuesto en el cuadro 4 refleja únicamente los aspectos que describen directamente el rol de los estudiantes en relación con el concepto de RSU identificados en nuestra investigación. Lo anterior, porque dentro de los resultados globales de nuestro estudio, además se identifican otras dos categorías del concepto de RSU: Generación y transmisión de conocimiento socialmente pertinente, y Gobierno y gestión universitaria responsable. En las categorías complementarias señaladas anteriormente, el rol de los estudiantes es menos relevante en el caso estudiado. 
CUADRO 4: CATEGORÍAS DE ANÁLISIS DE LOS RESULTADOS

\begin{tabular}{|c|c|c|c|}
\hline Códigos & Conceptos & Categorías & Categoría central \\
\hline Sensibilidad social & & \multirow{4}{*}{$\begin{array}{l}\text { Proceso } \\
\text { formativo } \\
\text { relacionado } \\
\text { con el } \\
\text { entorno. }\end{array}$} & \multirow{6}{*}{$\begin{array}{l}\text { Concepto de } \\
\text { responsabilidad } \\
\text { social universitaria. }\end{array}$} \\
\hline $\begin{array}{l}\text { Organizaciones } \\
\text { sociales } \\
\text { estudiantiles }\end{array}$ & $\begin{array}{l}\text { Voluntariado } \\
\text { universitario. }\end{array}$ & & \\
\hline $\begin{array}{l}\text { Formación } \\
\text { profesional }\end{array}$ & & & \\
\hline $\begin{array}{l}\text { Estrategias } \\
\text { enseñanza- } \\
\text { aprendizaje }\end{array}$ & Servicio. & & \\
\hline $\begin{array}{c}\text { Tramitación } \\
\text { personalidad } \\
\text { jurídica }\end{array}$ & \multirow{2}{*}{$\begin{array}{l}\text { Emprendimiento } \\
\text { Social. }\end{array}$} & \multirow{2}{*}{$\begin{array}{l}\text { Política } \\
\text { institucional } \\
\text { de vinculación } \\
\text { con el medio. }\end{array}$} & \\
\hline $\begin{array}{c}\text { Empleabilidad } \\
\text { titulados }\end{array}$ & & & \\
\hline
\end{tabular}

Fuente: elaboración propia.

En virtud de lo anterior, uno de los hallazgos interesantes de nuestra investigación son los procesos de voluntariado universitario que desarrollan los estudiantes en la ciudad de Antofagasta. Así, las actividades de voluntariado de los estudiantes universitarios les permite fortalecer su sensibilidad social, aun cuando formalmente no integran las mallas curriculares ni son expresión de alguna estrategia de enseñanza-aprendizaje utilizada por los docentes:

...es una necesidad generar un trabajo en la gente con la que tú trabajas, no puedes quedarte tranquilo al ver una realidad o una injusticia social, llegas al hecho que ya no puedes quedarte tranquilo, con el hecho de ver esa realidad social o injusticia social, y que quieres ser un ente de cambio frente a esa injusticia social, participar de ese cambio y no solamente donar el dinero una vez e irte o hacer un operativo e irte. Nosotros apuntamos a algo más, que ellos mismos lideren proyectos, que ellos mismos lideren cambios donde ellos trabajen... (Entrevista 32, dirigente estudiantil universitario).

...que consideren que también dentro de nuestra carrera se pueden desenvolver de otra forma, que a nosotros nos hablan mucho de que todos los conocimientos los tenemos que llevar a la práctica, la teoría lleva a la práctica, y queremos que también ellos apliquen todo su conocimiento en este tipo de trabajos, no solamente quizás de enfermería que todos conocen, también esta relación de ayuda, esa relación humana del ayudar al otro desconsideradamente, que no te importe recibir algo a cam- 
bio, eso es lo que queremos nosotros desarrollar en nuestros compañeros que se motiven de esa forma, pero no queremos que sea una parte de una asignatura, no queremos obligarlos a eso, eso nace en cada uno... (Entrevista 38, dirigente estudiantil universitario).

Por ello, es importante destacar que las acciones de voluntariado universitario para el caso de las universidades de la ciudad de Antofagasta, tienen diferentes puntos de partida que han impulsado estas actividades:

...emerge de manera intencional desde los propios alumnos, es decir, si no sabes tú hay una cantidad interna de grupos bien organizados sobre todo en el área de salud que, chicos que se programan muy bien y hacen un aporte efectivo de una vinculación ganar-ganar con el entorno, está: Odonto por ti, esta kinesiólogos en Acción, está Matronas en movimiento... (Entrevista 12, directivo universitario).

...nosotros partimos con el tema de la responsabilidad social y empezamos a capacitar a nuestros voluntarios, a formarlos y a hacer un trabajo mucho más profesional principalmente en campamentos, nosotros trabajamos mucho en hogares de menores y campamentos... (Entrevista 15 , directora de unidad universitaria).

De esta manera, el voluntariado universitario habitualmente es gestionado por la propia universidad a nivel institucional, pero en el caso específico de la Universidad de Antofagasta han sido los propios estudiantes los que han generado iniciativas de voluntariado para sus respectivas carreras:

...Odonto junto a ti comenzó el año 2009, producto de un grupo de alumnos de la carrera de odontología que se juntaron con el objetivo de fortalecer la formación del alumnado, tanto potenciar su actividad tanto en el área social, en el área culturales y en el área de investigación, la idea era generar un alumnado que tuviera mayores capacidades, esto debido a que nosotros en nuestra carrera nos forman netamente como dentistas no como el proceso universitario que debería ser el completo... (Entrevista 32, dirigente estudiantil universitario).

...Salud en terreno nace hace más menos ocho años, junto con la carrera de Medicina, nace con una iniciativa, desde los mismos alumnos, un poco con el objetivo de retomar el rol social que debe tener el médico, nuestra carrera ha sido un poco víctima del sistema, que se ha vuelto muy comercial... (Entrevista 33, dirigente estudiantil universitario). 
...nosotros lo que hacíamos era como llevar nuestros conocimientos tanto prácticos-teóricos a ellos que era la parte respiratoria y la parte estimulación temprana o sicomotricidad, pero más que nada estimulación temprana por que se refiere a un año, el 2012, bueno eso quedó el 2011 y ahí quedo todo ya, en ese tiempo se llamaba operativos kine, ahí no teníamos el nombre, y el 2012 como que tomamos más fuerza, entonces empezaron a preguntar qué nombre puede tener ya como más personalizándola y ahí dijeron ya Kinesiólogos en movimiento KEM... (Entrevista 40, dirigente estudiantil universitario).

Así, las organizaciones sociales estudiantiles «Odonto junto a ti», «Salud en terreno»y «Kinesiólogos en movimientos» se transforman en ejemplos del voluntariado propiciado y gestionado íntegramente desde y por los propios estudiantes, que ven en estos procesos una oportunidad muy importante para fortalecer su propio proceso de formación profesional, el cual a su juicio no les permite interactuar con la comunidad local y sus necesidades.

Las experiencias de voluntariado universitario descritas anteriormente permiten retomar el debate identificado en la literatura reseñada en este trabajo, respecto de si el voluntariado en la educación superior debe ser formal o informal, generado al alero institucional de la universidad o a partir de la creación y desarrollo de organizaciones especializadas en dichos temas, especialmente por la necesidad de una mayor flexibilidad administrativa que le proveen las organizaciones estudiantiles al voluntariado universitario:

...nuestra universidad lamentablemente tiene una serie de trabas burocráticas para poder hacer un trabajo en comunidad, por lo tanto nosotros nos vimos en la necesidad de trabajar de forma independiente en ciertos proyectos, por ejemplos realizar postulaciones a proyectos de fondos concursables, a través de la universidad es muy complicado, porque necesitas preguntarle hasta casi al rector, para poder hacerlo, tiene que pasar por toda la línea burocrática, ósea la Universidad, entonces nosotros decidimos tomar una vía aparte para hacer estos proyectos, lo que no quita que también trabajemos en conjunto, haciendo extensión por parte de la Universidad... (Entrevista 32, dirigente estudiantil universitario).

...ha sido bien complejo desde nuestra perspectiva como SET, la relación con la $\mathrm{U}$, porque nos costó mucho que nos reconocieran como agrupación, un poco porque existía la SEMUA que era más formal, que producía trabajos, organizan congresos, charlas que le benefician a la 
Universidad, a prestigio y estábamos nosotros, los desadaptados que íbamos a ver, que nos disfrazábamos de payasos, íbamos al hospital, que metíamos bulla, que hacíamos un par de cositas por aquí, un par de cositas por allá... (Entrevista 33, dirigente estudiantil universitario).

...que se preocupe de estas áreas y que las fomente, y las realce, porque por algo las otras agrupaciones tiene personalidad jurídica, y por algo el interés de ellos de no estar asociados con la universidad, y que no lo vean como algo negativo, sino que se hagan una introspección: que lo que están haciendo mal ellos... porque algo están haciendo mal para que sus propios alumnos estén descontentos, se separen y que no quieran trabajar todos juntos... (Entrevista 38, dirigente estudiantil universitario).

...hay grupos acá en la universidad que sí se vinculan con la gente, pero no son netamente creados por la universidad, por la institución son creados por los mismos alumnos, como son el Medicina en movimiento, los grupos de Kine que van a las poblaciones, por ejemplos acá hay niños que participan en el Adopta un Hermano, en el Techo, que se relaciona con la gente, pero no es un motivación plenamente de la universidad... (Entrevista 45, dirigente estudiantil universitario).

Es posible observar cierta tensión y crítica de los estudiantes hacia la universidad por algún tipo de aprovechamiento e instrumentalización de sus organizaciones de voluntariado, especialmente en el contexto del proceso de acreditación institucional de la universidad respecto del área de Vinculación con el medio:

...realmente era una gran suma para la universidad, hasta que vino el proceso de acreditación, ahí fue donde a través de la extensión, se nos... muestran horas, acreditan la extensión de la U, entonces gracias a eso, ocupan nuestra imagen para acreditar... (Entrevista 33, dirigente estudiantil universitario).

...no hay mucho apoyo, pero nosotras por ejemplo, lo que queremos ahora es oficializarla, y... Pero esto tantos pasos los que tenemos que hacer, te cuesta, mira lo de nosotros es una ayuda a la comunidad, pero finalmente a ellos igual les beneficia, porque... y a nuestra carrera igual le beneficia, y a la universidad le ayuda por lo que tiene que ver la acreditación. Entonces, yo digo, si ellos, a ellos también les sirve, deberían abrirnos más las puertas para que estas cosas se desarrollen dentro de la universidad, no solo con nuestra carrera, sino con todas... (Entrevista 38 , dirigente estudiantil universitario). 
Lo anterior, permite destacar que uno de los indicadores distintivos del voluntariado de los estudiantes de la Universidad de Antofagasta es que son los propios alumnos los que gestionan este proceso, mediante la tramitación de personalidad jurídica de sus organizaciones de voluntariado lo que se transforma en una clara demostración de encaminarlas hacia procesos de emprendimiento social:

...nuestro objetivo con la personalidad jurídica, fue que cualquier alumno que quisiera realizar un proyecto, pueda utilizar la personalidad jurídica para realizarlo, por eso entregar una herramienta, si alguien quiere organizar un campeonato deportivo, lo haga, es para el crecimiento como alumno, tiene que hacer, es parte de su formación como universitario. Si alguien quiere hacer un proyecto cultural que lo haga, tal vez nosotros no estemos trabajando directamente en eso, porque estamos en otras cosas, pero si lo puede hacer lo apoyamos para que lo haga, tiene que llevarlo a cabo... (Entrevista 32, dirigente estudiantil universitario).

...fueron gestiones propias, conseguimos financiamiento, la universidad también nos pasó un poco, unos doctores nos pasaron otro poco, nos movimos con unos laboratorios, siempre por las nuestras. Esta el proyecto también, el proyecto de sacar la personalidad jurídica, poder postular a este tipo de proyectos más grandes, pero ha sido difícil, un proceso complejo un poco por que el tiempo que tenemos no es mucho, nuestra carrera, también se caracteriza por eso, que es muy demandante de tiempo... (Entrevista 33, dirigente estudiantil universitario).

...eso es lo que igual queremos este año tratar de sacarla, porque ya se nos, si siguen entrando más voluntarios y si sigue entrando más gente, una agrupación así nomás, sin ingresos, sin formulación de proyecto se va a quedar en nada, al menos con una, desde mi punto de vista, con una personalidad jurídica se va hacer más responsabilidad de los voluntarios y de las personas que estén a cargo en el momento, porque si no como que lo ven como algo así no más, no le ven tanto, no le dan tanta importancia, a lo que se va un proyecto, así que la idea es sacar una personalidad jurídica para potenciar más a Nutrición a tu alcance... (Entrevista 34 , dirigente estudiantil universitario).

...nosotros ahora somos una organización social, necesitamos sacar personalidad jurídica para poder optar a distintos proyectos y así poder seguir creciendo. Entonces cual es como nuestro objetivo a largo plazo, es ser una corporación, entonces para llegar a eso, vamos a... necesitamos como el directorio que es como las personas que se encargan más como de las partes formal y tenemos la directiva, que ellos se encargan princi- 
palmente de los alumnos, de organizarnos... (Entrevista 40, dirigente estudiantil universitario).

La obtención de la personalidad jurídica le permite a las organizaciones de voluntariado universitario desarrollar las actividades de manera autónoma, sin la necesidad de depender de la universidad tanto administrativa como financieramente, teniendo la posibilidad de presentar proyectos para obtener financiamiento o establecer convenios de colaboración con otras instituciones. Finalmente, según algunos de los entrevistados el voluntariado universitario no tiene impactos únicamente en el ámbito formativo, sino que también puede potenciar la empleabilidad de los titulados al ser considerado como un plus en los antecedentes a la hora de postular a un puesto de trabajo:

...los jóvenes que nosotros logramos tener al interior de la Pastoral que reciben la formación nuestra, que se capacitan que hacen todo con nosotros, si hemos tenido la alegría de que se han recibido y que profesionalmente por un lado le han considerado eso, porque ellos los ponen en su currículo todas las capacitaciones, entonces hay muchas empresas y muchas instituciones estatales que están considerando eso... (Entrevista 15, directora de unidad universitaria).

...lo sé por estudios de Trabajando.com, esta empresa de Santiago que se dedica como a hacer una bolsa de trabajo, que hoy día es un plus en el currículo de las personas poner que hacen voluntariados [...] en Inglaterra si tú no poni en tu currículo que hiciste voluntariado es como que pusiera hoy día que no hablas inglés, yo siento que las universidades se están dando cuenta de eso... (Entrevista 24, ONG).

...en otros países, por ejemplo, se valora mucho que tu tengas voluntariado. Entonces ¿qué pasa? El cabro que está estudiando sabe que si no sale de la universidad con un voluntariado o dos voluntariados o tres voluntariados, le va a costar el doble de tiempo encontrar pega a que si lo tiene... (Entrevista 25, empresa).

La capacidad del voluntariado de potenciar las posibilidades de mayor empleabilidad de los titulados universitarios descrita en las entrevistas citadas anteriormente, es un valor agregado importante para el desarrollo de este tipo de instancias al interior de las universidades de la ciudad de Antofagasta, por lo que la universidad socialmente responsable debiera potenciar y apoyar el desarrollo de este tipo de iniciativas, incluso si se generan por motivación de los propios estudiantes. 
Así, el voluntariado universitario en el caso de las universidades de la ciudad de Antofagasta, se plantea primero a partir de una iniciativa e interés propio de un grupo importante de estudiantes de diferentes carreras e incluso de diferente universidad. No obstante, los estudiantes desarrollan el voluntariado asociado a sus propias carreras, poniendo sus conocimientos y competencias a disposición de la comunidad o de grupos más vulnerables, lo que a la vez les permite desarrollar procesos de aprendizaje servicio.

$\mathrm{Al}$ comparar con otras investigaciones, podemos mencionar el estudio que desarrollan Jara y Vidal (2010) con estudiantes de educación superior de la Provincia de Concepción en Chile, donde se logra establecer que los estudiantes que desarrollan voluntariado obtuvieron altos puntajes en responsabilidad social como conducta previa a la experiencia como voluntarios, expresado en cuestiones tales como ejercicio profesional más comprometido y formación de conciencia social.

Asimismo, los propios Jara y Vidal (2010) plantean que debe estudiarse cuáles podrían ser los factores que generan una mayor responsabilidad social en estos estudiantes antes de ser voluntarios, lo que podría deberse a variables no observables sino que más a aspectos intrínsecos propios de cada estudiante voluntario, por lo que el voluntariado en este caso solo reafirmaría la alta responsabilidad social preexistente en ellos.

Para finalizar el análisis sobre el voluntariado universitario, resulta interesante citar una de las conclusiones del estudio de Ríos (2004), quien analiza el involucramiento de estudiantes de la Pontificia Universidad Católica de Chile en acciones filantrópicas, donde señala que «el voluntariado de los estudiantes universitarios debe ser considerado no sólo como parte de su formación personal sino también de la preparación profesional por las universidades. En consecuencia, deben procurar oportunidades, recursos y reconocimiento académico a este tipo de actividad. Sin embargo, este mayor énfasis profesional debe hacerse enfatizando la donación de sí mismo, la caridad, por lo que la formación valórica no sólo no debería descuidarse, sino, al contrario, reforzarse». Allí se resume de manera bastante precisa lo que las universidades de la ciudad de Antofagasta deben hacer en el contexto del fortalecimiento de su comportamiento socialmente responsable, específicamente en lo relativo al voluntariado universitario. 


\section{CONCLUSIONES}

La experiencia de los estudiantes relativa al voluntariado universitario descrita en este trabajo identifica importantes posibilidades de interacción con el aprendizaje servicio, específicamente como expresión de servicio en beneficio de la comunidad lo que les permite a los alumnos fortalecer sus conocimientos y desarrollar competencias de prosocialidad y compromiso ciudadano. Lamentablemente, las universidades a las que pertenecen los jóvenes voluntarios del caso estudiado no ofrecen espacios de validación institucional/académica a estas actividades, traducida por ejemplo en el reconocimiento de créditos dentro de su formación como resultado del desarrollo de actividades complementarias, o que se concrete en un apoyo económico para el desarrollo de dichas acciones de voluntariado.

En cambio, si existe un mayor reconocimiento institucional por parte de las universidades incluidas en el caso de estudio respecto del voluntariado estudiantil en lo relativo a su consideración como actividad de vinculación con el medio, en el contexto de los procesos de acreditación institucional. Dicha situación es muy criticada por los dirigentes estudiantiles entrevistados durante la investigación, reconociéndola como una clara práctica de instrumentación de sus iniciativas de voluntariado universitario.

La dicotomía expuesta anteriormente en cuanto a la ambivalencia de las universidades frente al voluntariado de los estudiantes, requiere de una importante revisión y reconsideración por parte de las instituciones universitarias estudiadas, especialmente en el contexto del concepto de RSU en el cual se desarrolla la investigación a la que pertenece este artículo. Lo anterior, fundamentalmente porque no pareciera ser una práctica muy socialmente responsable el que por un lado los estudiantes no tengan mucho respaldo económico ni reconocimiento docente para el voluntariado que desarrollan, el que sí es reconocido instrumentalmente para efectos de la acreditación institucional de las universidades, situando el concepto de RSU para el caso estudiado como algo más próximo a las teorías instrumentales del comportamiento socialmente responsable descritas por Garriga y Melé (2004) para el ámbito empresarial, o de tipo gerencial-directiva identificada por Gaete (2011) para el ámbito de la RSU. 
Otro aspecto valioso de la experiencia de voluntariado universitario desarrollado por los alumnos de la ciudad de Antofagasta, es su capacidad de conectarlo con incipientes procesos de emprendimiento social, especialmente dada la enorme habilidad de gestión y compromiso social demostrada por los estudiantes que participan en las organizaciones de voluntarios, muy especialmente de sus dirigentes quienes vienen desarrollando estas iniciativas con motivo del movimiento estudiantil que mantuvo tomadas las universidades de la ciudad por varios meses el año 2011. Por esto, el potencial emprendedor que demuestran sus proyectos de voluntariado universitario, sumado al positivo impacto de dichas prácticas en su propia formación como espacio de aprendizaje servicio, permiten identificar elementos que lo separan de las expresiones o miradas más tradicionales de carácter filantrópico con las que muchas veces es desarrollado el voluntariado en diferentes contextos sociales, logrando que sus iniciativas sean más sustentables en el tiempo cuando las enfocan como un emprendimiento social.

De esta manera, uno de los aspectos tremendamente destacables de la experiencia del voluntariado universitario de la ciudad de Antofagasta es la enorme coherencia y conciencia social con la cual sus líderes han visionado estos proyectos, buscando contribuir desde su formación y participación como estudiantes universitarios a las diversas problemáticas existentes actualmente en la sociedad antofagastina. Lo anterior, es especialmente observable en las carreras del área de la salud, respecto de personas con importantes niveles de vulnerabilidad social, lo que le asigna a estas experiencias un valor e impacto en la sociedad aun mayor, traduciéndose incluso en el interés de instituciones públicas como el Servicio de Salud de Antofagasta para asociarse con las organizaciones estudiantiles de voluntariado, con el propósito de desarrollar acciones conjuntas en materia de sensibilización e incluso diagnóstico de algunas patologías básicas, situación que ha motivado a los estudiantes a transformar su voluntariado universitario en un emprendimiento social.

Así, la universidad socialmente responsable debe asumir como uno de sus desafíos estratégicos relevantes al desarrollo de acciones que permitan articular al aprendizaje servicio, el voluntariado universitario y el emprendimiento social como instancias permanentes dentro del quehacer universitario, beneficiando con ello a sus propios estudiantes pero también al resto de la comunidad, lo cual debe traducirse en acciones de reconocimiento académico y recursos económicos que apoyen a los estudiantes en la ejecución y consolidación de su 
voluntariado, situación que lamentablemente no ocurre de manera adecuada en el caso estudiado.

ANTOFAGASTA (CHILE), DICIEMBRE 2014

RECIBIDO: DICIEMBRE 2014

ACEPTADO: JUNIO 2015

\section{REFERENCIAS BIBLIOGRÁFICAS}

ALONSO, LUIS (2003): La mirada cualitativa en sociología. Madrid: Fundamentos.

ARIAS, SILVIA (2008): Voluntariado universitario. Guía para su gestión en las universidades madrileñas. Madrid: Dirección General de Voluntariado y Promoción Social.

ARRATIA, ALEJANDRINA (2008): «Ética, solidaridad y aprendizaje servicio en la educación superior». Acta Bioethica, Vol.14, $\mathrm{N}^{\circ} 1$.

BARDIN, LAURENCE (2002): Análisis de contenido. Madrid: Akal.

BARGSTED, MARIANA (2013): «El emprendimiento social desde una mirada psicosocial». Revista Civilizar, Vol.13, ํ2ㄴ.

BAtLle, Roser (2009): «El servicio en el aprendizaje servicio». En JoseP PUIG (coordinador) y VV.AA: Aprendizaje Servicio (ApS). Educación y compromiso cívico. Barcelona: Graó.

BLANCO, ASCENSIÓN, AlberTo GaRín y ROSA ROdRÍGUEZ (2012): «Emprendimiento social: desarrollo de competencias y valores en el marco universitario». En ROSA RODRÍGUEZ (coordinadora): Educación en valores en el ámbito universitario. Propuestas y experiencias. Madrid: Narcea.

BRINGLE, ROBERT y JULIE HATCHER (1996): «Implementing Service Learning in Higher Education». The Journal of Higher Education, Vol. 67, №2.

Charmaz, KATHY (2006): Constructing Grounded Theory. A practical guide through qualitative analysis. London: SAGE.

Cicero, Sergio, José Asón y Alicia SANTAMaría (2012): 100 buenas prácticas en emprendimiento universitario. La Coruña: Netbiblo.

Corbetta, Piergiorgio (2003): Metodología y técnicas de investigación social. Madrid: Mc Graw Hill.

CURTO, MARTA (2012): «Los emprendedores sociales: innovación al servicio del cambio social». Cuaderno $\mathrm{N}^{\circ} 13$ de la Cátedra La Caixa de Responsabilidad social de la empresa y Gobierno corporativo. IESE-Universidad de Navarra.

FriRe, PAUlo (2008): Pedagogía del oprimido. Buenos Aires: Siglo Veintiuno. 
GAETE, RICARDO (2011): «La responsabilidad social universitaria como desafío para la gestión estratégica de la educación superior: el caso de España». Revista de Educación №355.

GARRIDA, EliSABET y DomÈnec Melé (2004): «Corporate Social Responsibility Theories: Mapping the Territory». Journal of Business Ethics, Vol. 53, $\mathrm{N}^{\mathrm{o}} 1-2$.

GATICA, SEBÁSTIÁN (2011): Emprendimiento e innovación social: construyendo una agenda pública para Chile. Santiago: Centro de Políticas Públicas UC.

GonZÁLEZ, JORGE y CARLOS LÓPEZ (2012): «El emprendimiento en los sistemas universitarios. El Tecnológico de Monterrey». Serie Políticas Públicas y Transformación Productiva $\mathrm{N}^{\circ} 12$. Corporación Andina de Fomento.

Gray, Maryann, Elizabeth OndaAtje, Ronald Fricker y Sandy GesCHWIND (2000): «Assessing Service-Learning Results from a Survey of Learn and Serve America, Higher Education». Change, Vol. 32. №2.

GuZMÁn, AleXANDER y MARÍA TRUjILlo (2008): «Emprendimiento social. Revisión de literatura». Estudios Gerenciales, Vol.24, №109.

JARA, KARINA y DAISY VIDAL (2010): «Voluntariado y responsabilidad social en jóvenes estudiantes de educación superior de la provincia de Concepción, Chile». Revista Trayectorias, Vol. 12, N³1.

LESTER, JESSICA, BOB KRONIEK y MARK BENSON (2012): «A University Joins the Community». The Phi Delta Kappan, Vol. 93, №6.

MARTín, NATALIA y VICENTE LiMA (2013): «La intención emprendedora de los estudiantes universitarios en diferentes sistemas educativos. El caso español versus el caso brasileño». En ESPERANZA GIL (coordinadora): Emprendimiento social y educativo: nuevos tiempos, nuevos retos. Madrid: ACEDE/Universidad Complutense.

MARTÍNEZ, MIQUEL (2010): «Aprendizaje servicio y construcción de ciudadanía activa en la universidad: la dimensión social y cívica de los aprendizajes académicos». En MIQUEL MARTíNEZ (editor): Aprendizaje servicio y responsabilidad social de las universidades. Barcelona: Octaedro.

MÉNDEZ, MARÍA (2009): «Voluntariado universitario, participación ciudadana y desarrollo». Ponencia presentada al IX Congreso Anual de investigación sobre el tercer sector en México.

Disponible en línea: www.lasociedadcivil.org.

MOONEY, LINDA y BOB EDWARDS (2001): «Experiential Learning in Sociology: Service Learning and Other Community-Based Learning Initiatives». Teaching Sociology, Vol. 29, №2.

PARKER-GWIN, RACHEL (1996): «Connecting Service to Learning: How Students and Communities Matter». Teaching Sociology, Vol. 24, №1.

PROGRAMA DE VOLUNTARIOS DE LAS NACIONES UNIDAS (2011): «V Informe sobre el estado del voluntariado en el mundo. Valores universales para alcanzar el bienestar mundial». Disponible en línea: www.unv.org. 
PUTNAM, ROBERT (editor) (2003): El declive del capital social. Un estudio internacional sobre las sociedades y el sentido comunitario. Barcelona: Galaxia Gutenberg.

RÍOS, RENÉ (2004): «Universitarios y voluntariado: análisis del involucramiento en acciones filantrópicas de los alumnos de la PUC». Psykhe, Vol. 13, No2.

RODRÍGUEZ, MARGARITA (2014): «El aprendizaje-servicio como estrategia metodológica en la universidad». Revista Complutense de Educación, Vol. 25, $\mathrm{N}^{\mathrm{o}} 1$.

RODRÍGUEZ, CARLOS y FrANK PRIETO (2009): «La sensibilidad al emprendimiento en los estudiantes universitarios. Estudio comparativo Colombia-Francia». Revista Innovar, Número Especial Educación.

SedlaK, CAROL, Margaret DoHENy, NANCY PANTHOFER y Ella ANAYA (2009): «Critical Thinking in Students Service-Learning Experiences». College Teaching, Vol. 51, N³.

Silva, YAmila; MARIEANEla CARENA y MARIO CANUTO (2013): «Niveles de engagement y burnout en voluntarios universitarios. Un estudio exploratorio y descriptivo». Boletín de Psicología $\mathrm{N}^{\circ} 108$.

SOLER, PATRICIA (2007): «Factores psicosociales explicativos del voluntariado universitario». Tesis Doctoral, Departamento de Sociología II, Psicología, Comunicación y Didáctica, Facultad de Económicas, Universidad de Alicante.

STAKE, ROBERT (2007): Investigación con estudio de casos. Madrid: Morata.

STRAUSS, ANSELM y JULIET CORBIN (2002): Bases de la investigación cualitativa. Técnicas y procedimientos para desarrollar la teoría fundamentada. Medellín: Contus.

TRILlA, JAUME (2009): «El aprendizaje servicio en la pedagogía contemporánea». En JOSEP PUIG (coordinador) y VV.AA: Aprendizaje Servicio (ApS). Educación y compromiso cívico. Barcelona: Graó.

VALLAEYS, FRANÇOIS (s/f): «El voluntariado solidario: ventajas y peligros». Disponible en línea: www.rsu.uninter.edu.

Werts, DAVID, Alberto CABrera y ThOMAS SANFORD (2010): «Beyond Giving: Political Advocacy and Volunteer Behaviors of Public University Alumni». Research in Higher Education, Vol. 51, №4. 